Short communication

\title{
O 026 - Negative impact of muscle weakness and spasticity on gait in children with unilateral cerebral palsy
}

\author{
E. Papageorgiou ${ }^{\mathrm{a}, *}$, C. Simon-Martinez ${ }^{\mathrm{a}}$, A. Van Campenhout ${ }^{\mathrm{b}, \mathrm{c}}$, K. Desloovere ${ }^{\mathrm{a}, \mathrm{d}}$ \\ a Katholieke Universiteit Leuven, Rehabilitation Sciences and Physiotherapy, Leuven, Belgium \\ ${ }^{\mathrm{b}}$ Katholieke Universiteit Leuven, Development and Regeneration, Pellenberg, Belgium \\ ${ }^{c}$ UH Leuven, Orthopaedics, Leuven, Belgium \\ ${ }^{\mathrm{d}}$ UH Leuven, Clinical Motion Analysis Laboratory, Pellenberg, Belgium
}

A R T I C L E I N F O

Keywords:

Cerebral palsy

Gait

Muscle weakness

Spasticity

Statistical parametric mapping

\section{Introduction}

Associations between muscle weakness or spasticity and gait of children with cerebral palsy (CP) exist $[1,2]$, but they are not always easily mapped. A regression analysis using statistical non-parametric mapping (SnPM), allows the exploration of these associations, over the entire gait cycle (GC) [3], by applying a corrected statistical threshold.

\section{Research question}

a) Can SnPM identify relationships between muscle weakness or spasticity at the level of a specific joint and the respective sagittal plane joint motion? b) Is each joint motion also influenced by the total lower limb muscle weakness or spasticity?

\section{Methods}

A retrospective sample of 114 children with unilateral CP ( $n=52$ left, GMFCS level $\mathrm{I}=92$, median age $10 \mathrm{y} 2 \mathrm{~m}$ ) was selected for this study, without any botulinum toxin injections in the last 6 months or lower limb surgery in the last 4 years. Two or 3 trials were averaged for further analysis ( $\mathrm{n}=3$ for 91 patients). A linear regression analysis (with $\mathrm{a}=0.01$ ) was used with the non-parametric version of SPM1d to investigate to what extent the kinematic waveforms of the sagittal plane motion of the pelvis, hip, knee or ankle were influenced by a) muscle weakness or spasticity at the level of a specific joint or b) the total lower limb muscle weakness or spasticity. Summed muscle weakness or spasticity scores of the main muscles were used to define the level of impairment for each joint or the total lower limb.

\section{Results}

a) All joint motions were influenced by muscle spasticity at the level of a specific joint during 7-44\% of the GC (Fig. 1), whereas only pelvis and ankle motions were influenced by weakness of the surrounding muscles ( $80 \%$ and $15 \%$ of the GC respectively, Fig. 2). b) Total lower limb spasticity impacted all joint motions, whereas no significant association between total lower limb weakness and hip motion in the sagittal plane was found (Table 1).

\section{Discussion}

$\mathrm{SnPM}$ is able to map the negative influence of muscle weakness and spasticity on the sagittal plane motion of children with unilateral $\mathrm{CP}$, while considering the entire GC and avoiding a "regional focus bias" [4]. This investigation confirms the negative impact of muscle weakness or spasticity at the level of each individual joint reported by previous studies [1,2], while simultaneously providing first evidence on the negative impact of the composite score of total lower limb impairments on each joint motion. Future research should explore the influence of selective motor control as well as the combined influence of all neuromotor impairments on $\mathrm{CP}$ gait.

\footnotetext{
* Corresponding author.

E-mail address: eirini.papageorgiou@kuleuven.be (E. Papageorgiou).
} 

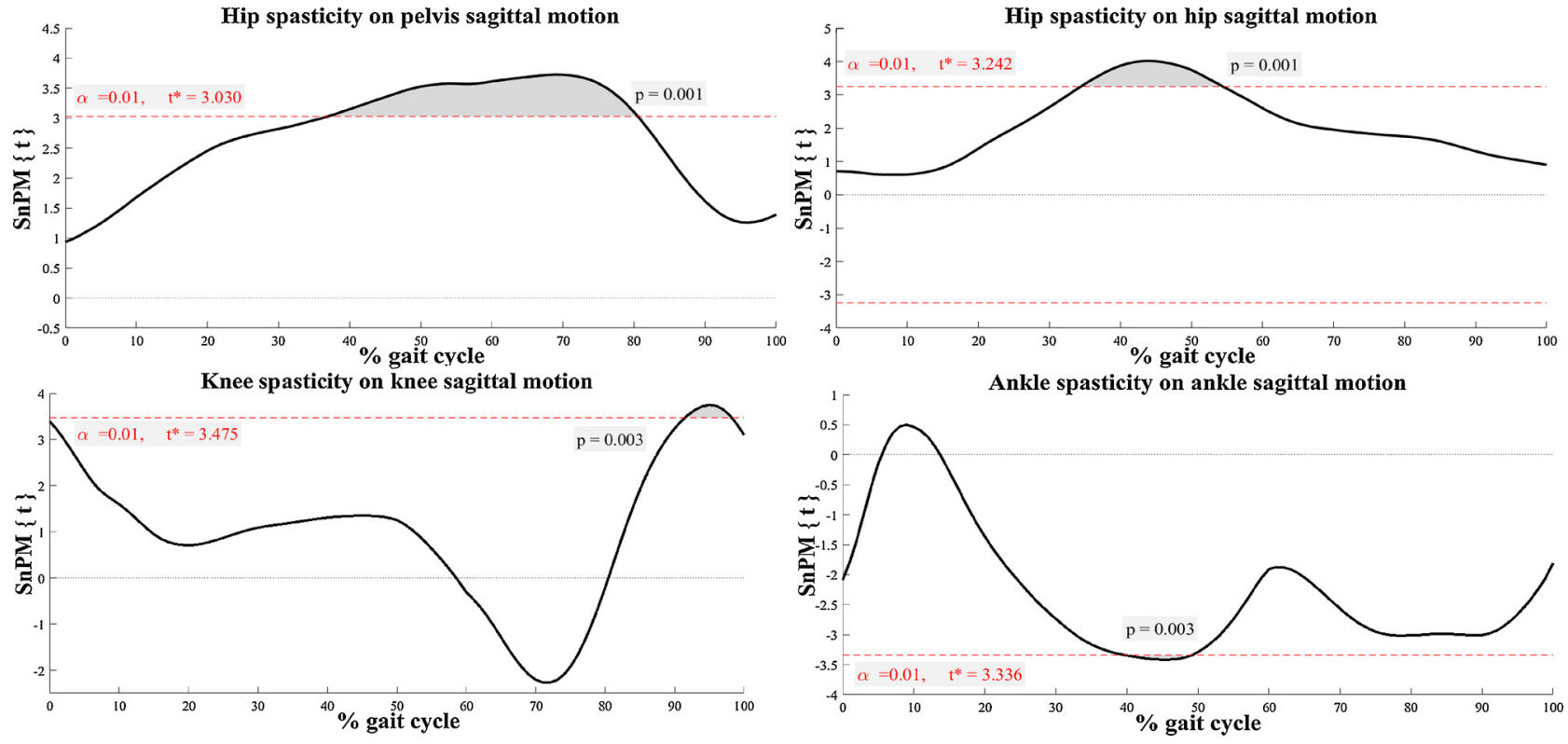

Fig. 1. Influence of muscle spasticity at the level of a specific joint on respective sagittal plane joint motion.
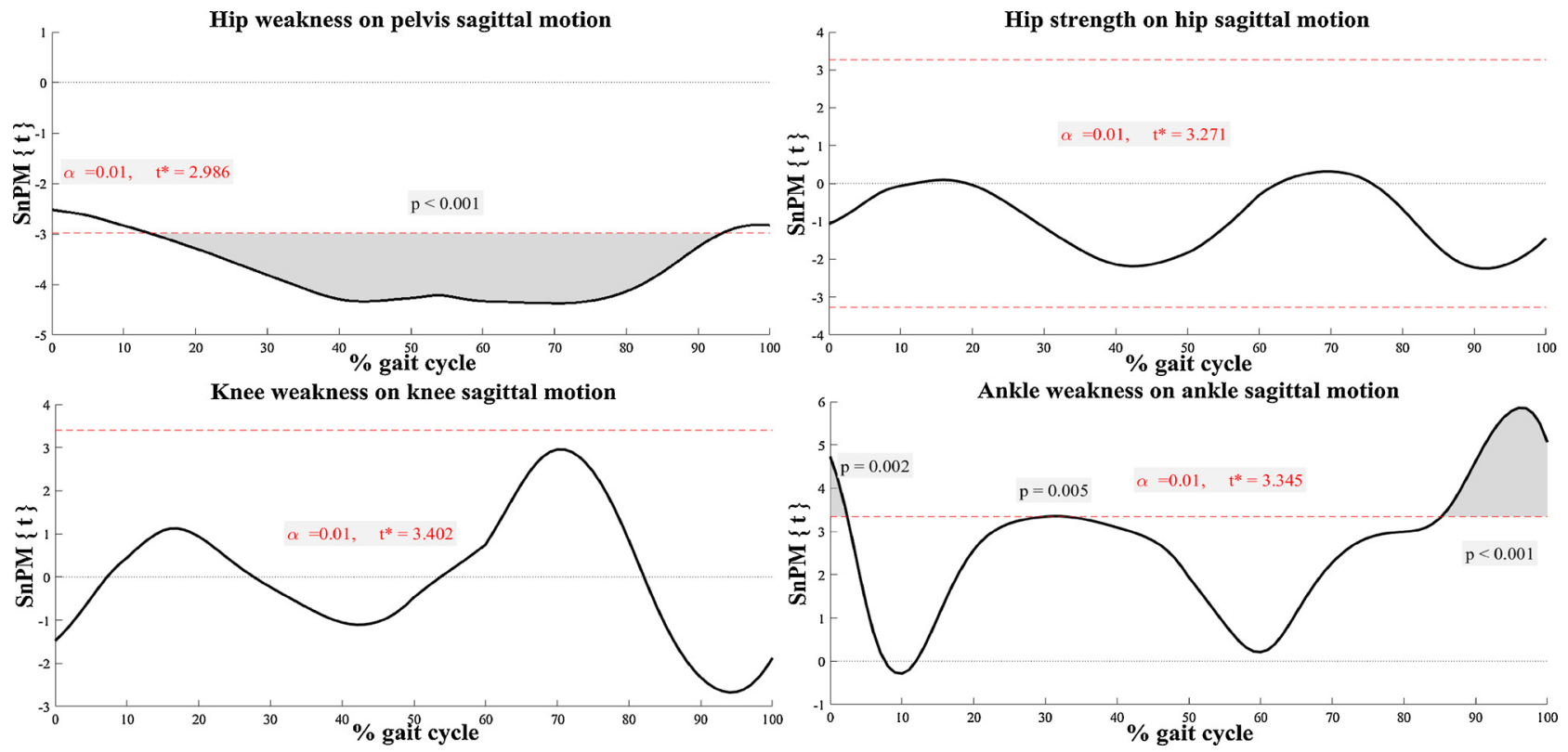

Fig. 2. Influence of muscle weakness at the level of each joint on respective sagittal plane joint motion.

Table 1

Influence of total spasticity and weakness on sagittal plane motion of pelvis, hip, knee and ankle.

\begin{tabular}{|c|c|c|c|c|c|c|c|c|c|c|}
\hline & \multicolumn{5}{|c|}{ Total spasticity } & \multicolumn{5}{|c|}{ Total weakness } \\
\hline Pelvis & 1 & 0.00001 & 75.70 & {$[12,0187,71]$} & 3.0202 & 1 & 0.00002 & 59.44 & {$[26,82 \quad 86,26]$} & -3.0178 \\
\hline \multirow[t]{2}{*}{ Knee } & 1 & 0.0033 & 5.39 & {$[91,2496,63]$} & 3.4474 & 2 & 0.00001 & 14.88 & {$[59,5274,40]$} & 3.3643 \\
\hline & & & & & & & 0.00001 & 13.76 & {$[85,72$ 99,48] } & -3.3643 \\
\hline \multirow[t]{2}{*}{ Ankle } & 1 & 0.0032 & 6.81 & {$[88,3295,13]$} & -3.2318 & 2 & 0.0035 & 1.10 & {$\left[\begin{array}{ll}0 & 1,10]\end{array}\right.$} & 3.2431 \\
\hline & & & & & & & 0.00004 & 12.95 & {$[87,05100]$} & 3.2431 \\
\hline
\end{tabular}

Total spasticity: sum of spasticity of hip flexros, hip adductors, hamstrings, rectus femoris, soleus, gastrocnemius and tibialis posterior; Total weakness: sum of weakness of hip flexors / extensors / abductors / adductors, knee flexors / extensors, ankle dorsi- / plantarflexors; Clusters: areas where SnPM $\{\mathrm{t}\}$ statistic crosses the critical threshold ( $\left.t^{*}\right)$; Extent: duration of the identified cluster; Endpoints: beginning and end point of the identified cluster; $t^{*}$ : critical threshold needed to be crossed for SnPM to identify significant areas of association between total spasticity or weakness and sagittal plane motion of pelvis, hip, knee and ankle. 


\section{References}

[1] K. Desloovere, G. Molenaers, H. Feys, C. Huenaerts, B. Callewaert, P. Van de Walle, Do dynamic and static clinical measurements correlate with gait analysis parameters in children with cerebral palsy? Gait Posture 24 (3) (2006) 302-313, http://dx.doi. org/10.1016/j.gaitpost.2005.10.008.

[2] A. Nieuwenhuys, E. Papageorgiou, S.-H. Schless, T. De Laet, G. Molenaers,

K. Desloovere, Prevalence of joint gait patterns defined by a Delphi consensus study is related to gross motor function, topographical classification, weakness, and spasticity, in children with cerebral palsy, Front. Hum. Neurosci. 11 (185) (2017), http://dx.doi.org/10.3389/fnhum.2017.00185.

[3] T.C. Pataky, One-dimensional statistical parametric mapping in Python, Comput. Methods Biomech. Biomed. Eng. 15 (3) (2012) 295-301, http://dx.doi.org/10.1080/ 10255842.2010 .527837$.

[4] T.C. Pataky, M.A. Robinson, J. Vanrenterghem, Vector field statistical analysis of kinematic and force trajectories, J. Biomech. 46 (14) (2013) 2394-2401, http://dx. doi.org/10.1016/j.jbiomech.2013.07.031. 